\title{
Colgajo keystone. Una opción cuando el cierre primario no es posible
}

\section{Keystone flap. An option when the primary closure is not possible}

\author{
Juan Castro-Ayala ${ }^{1 *}$, Gastón Galimberti², Julio Parra-Cares ${ }^{1}$, Leisa Molinari² y Damián Ferrario² \\ ${ }^{1}$ Departamento de Dermatología, Pontificia Universidad Católica de Chile, Santiago, Chile; ${ }^{2}$ Servicio de Dermatología, Centro de Cáncer de Piel y \\ Cirugía Micrográfica de Mohs, Hospital Italiano de Buenos Aires, Buenos Aires, Argentina
}

\begin{abstract}
Resumen
La reconstrucción de defectos posoperatorios $>15 \mathrm{~mm}$, ubicados en áreas con escasa movilidad o piel, puede representar un desafío. Algunas de estas áreas incluyen rodillas, piernas, pies, hombros, antebrazos y manos. El cierre primario puede no ser recomendable o posible, lo cual establece la necesidad de aplicar colgajos o injertos, estos últimos con resultados estéticos desfavorables. El colgajo fasciocutáneo keystone (CK) es una opción relativamente fácil para reparar defectos en estas regiones, con un período posoperatorio de recuperación rápido y resultados estéticos muy aceptables. El objetivo de este artículo es revisar paso a paso la técnica, indicaciones, ventajas y posibles complicaciones.
\end{abstract}

Palabras claves: Colgajo keystone. Colgajo quirúrgico. Colgajo fasciocutáneo. Defectos cutáneos.

\section{Abstract}

Reconstruction of greater than $15 \mathrm{~mm}$ postoperative defects, located in areas with poor mobility or skin, can be a challenge. Some of these areas include knees, legs, feet, shoulders, forearms and hands. Primary closure may not be advisable or possible, establishing the need of flaps or grafts; latter with unfavorable aesthetic results. Keystone fasciocutaneous flap (CK) is a relatively easy option for to repair defects in these regions, with a rapid recovery postoperative period and very acceptable aesthetic results. The objective of this article is to review the technique step by step, indications, advantages and possible complications.

Key words: Keystone flap. Surgical flap. Fasciocutaneous flap. Skin defects.

\section{Introducción}

En el año 2003, Behan descrito el colgajo fasciocutáneo en piedra clave (keystone [CK]) como un colgajo en isla en $\mathrm{V}-\mathrm{Y}$ con forma trapezoidal (Fig. 1A-B). Debe su nombre a la similitud con las piedras romanas que soportaban el peso de los arcos 1 .

Es una alternativa para los injertos cutáneos y otros colgajos empleados para cubrir y reconstruir defectos posquirúrgicos $>15 \mathrm{~mm}$, ubicados en áreas
Disponible en internet: 01-09-2021 Med Cutan Iber Lat Am. 2021;49(3):132-136 www.MedicinaCutanealLA.com licencia CC BY-NC-ND (http://creativecommons.org/licenses/by-nc-nd/4.0/) 


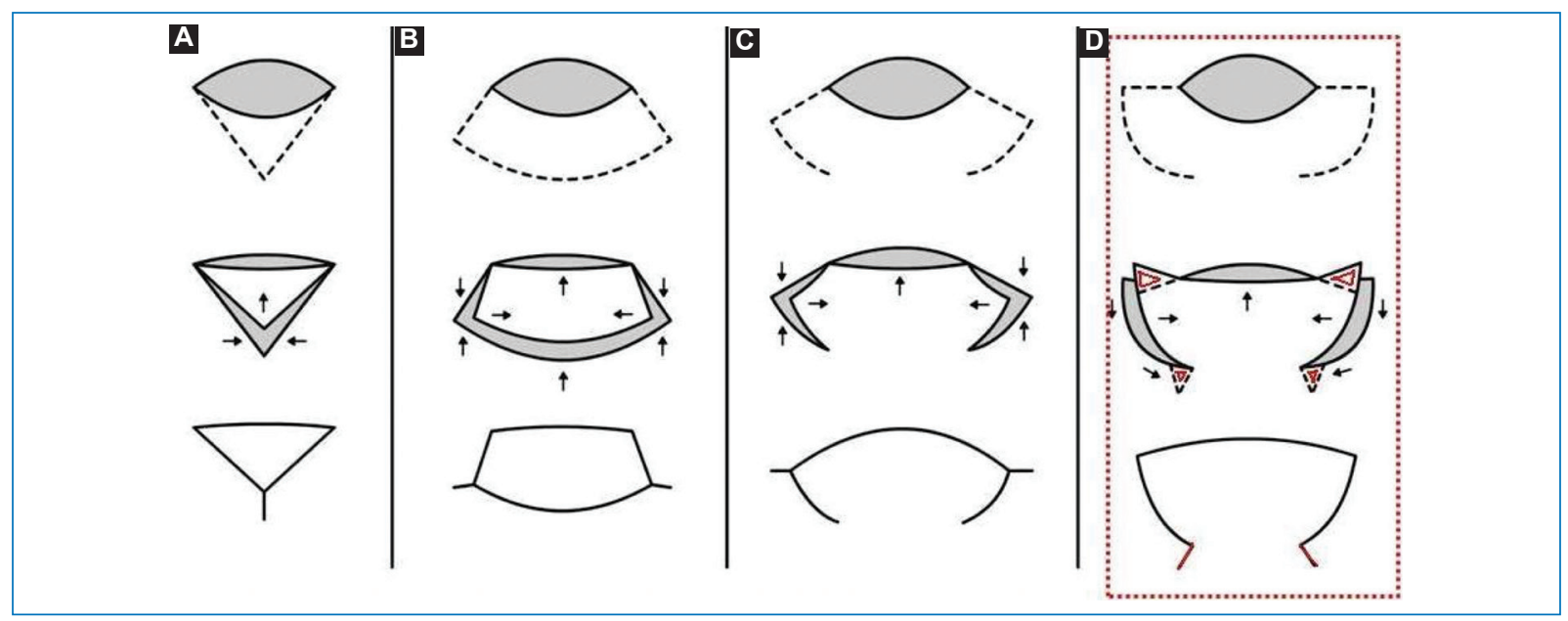

Figura 1. Distintas posibilidades de diseño. A-B: diseño original de colgajo en isla y colgajo keystone de forma trapezoidal. C-D: modificaciones del colgajo keystone en.

con poca movilidad o escasez de piel sobrante donde el cierre primario puede no ser aconsejable o posible. Se trata de un colgajo fasciocutáneo cuya vascularización depende de las arteriolas perforantes musculares que ofrece una ventaja técnica y cosmética, dado que permite el cierre en un acto quirúrgico único (defecto y zona donante), sin precisar autoinjertos cutáneos, cuyos resultados estéticos son poco favorables ${ }^{2-4}$.

Las modificaciones en el CK han incrementado la magnitud de la cobertura de tejidos blandos de grandes defectos. Una de esas modificaciones es el CK con pedículo cutáneo (Fig. 1C-D), que no compromete la irrigación del colgajo, con menor riesgo de necrosis y congestión linfática y venosa ${ }^{5-7}$.

Se describe a continuación la técnica secuencial (con esquema y registro iconográfico), junto con indicaciones, ventajas y posibles complicaciones (Fig. 2).

\section{Técnica quirúrgica}

\section{Primer paso: diseño del colgajo (Fig. 2A)}

Para simplificar el diseño del colgajo se toman como referencia tres puntos numerados del 1 al 3 . El punto 1 se marca en la mitad del borde lateral del defecto. De este punto 1 se traza una línea recta equivalente a 1 a 1.5 veces el largo del defecto original y se arriba al punto 2. La angulación de ese trazado puede variar de $90^{\circ}$ a $135^{\circ}$ (Fig. 1C-D).

Desde el punto 1 se traza una línea imaginaria de al menos el mismo tamaño del ancho del defecto y se marca el punto 3 (convergencia contralateral). Luego se unen en forma de semicírculo los puntos 2 y 3 . Se repiten estos pasos en espejo contralateral y se obtiene el diseño final del colgajo.

\section{Segundo paso: incisión de la piel (Fig. 2B)}

Se efectúa la incisión de piel de acuerdo con el diseño. Se debe incluir todo el espesor del tejido celular subcutáneo (en algunas zonas como la rodilla y el dorso de la mano es muy escaso). Se realiza la divulsión por encima de la fascia muscular en las regiones laterales. Por debajo del colgajo se deja una zona sin divulsión y de esa forma se logra un colgajo fasciocutáneo en isla con perforantes que suministren la irrigación. Si se requiere mayor movilización, se realiza una tunelización con tijera de disección de punta roma en la zona donde se encuentra el pedículo cutáneo. Esto le confiere mayor movimiento al colgajo para el cierre del defecto.

\section{Tercer paso: avance y fijación (Fig. 2C)}

El colgajo se avanza y se cubre el defecto. Se fija con puntos en polea de nailon en el sitio de mayor tensión. Se sugiere crear puntos externos cruzados en X (Fig. 2D) para completar el cierre del defecto. Los puntos se inician desde sitios de menor tensión hasta los de mayor tensión, lo cual permite que las fuerzas se distribuyan mejor a lo largo de todo el colgajo. 


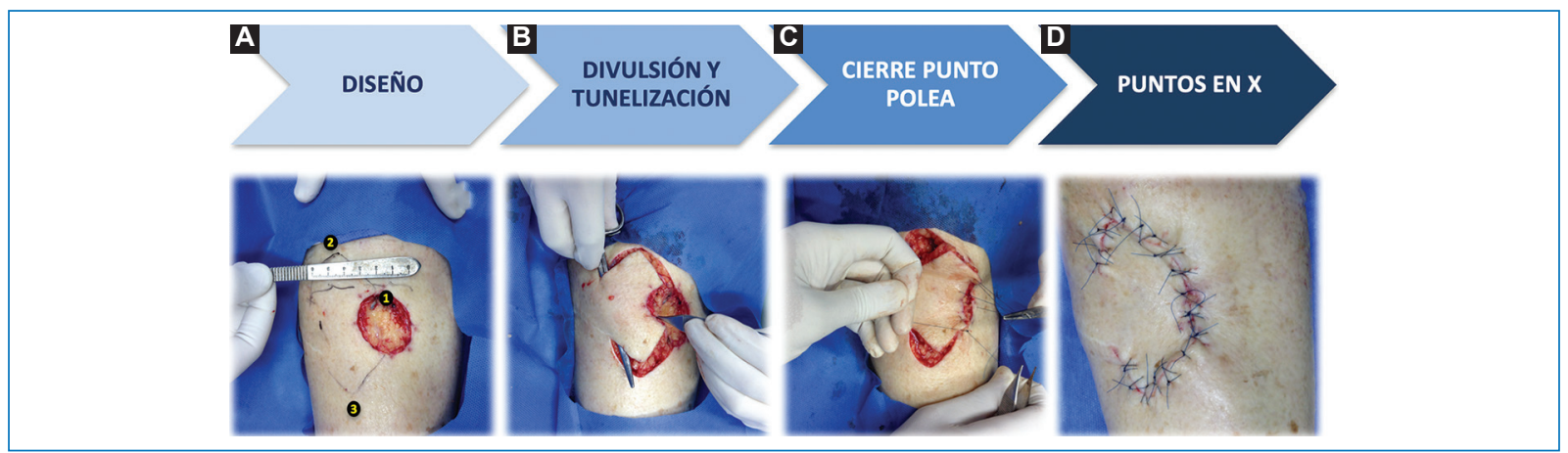

Figura 2. Esquema del diseño y ejecución quirúrgica. A: diseño del colgajo con uso de mango de bisturí métrico; unión de los puntos 1, 2 y 3. B: esquema de la divulsión por debajo del tejido celular subcutáneo y tunelización con tijera de bordes romos para formar un puente entre el colgajo y el pedículo y mejorar su movilidad. C: fijación de puntos de mayor tensión (puntos polea). D: puntos discontinuos cruzados en X.

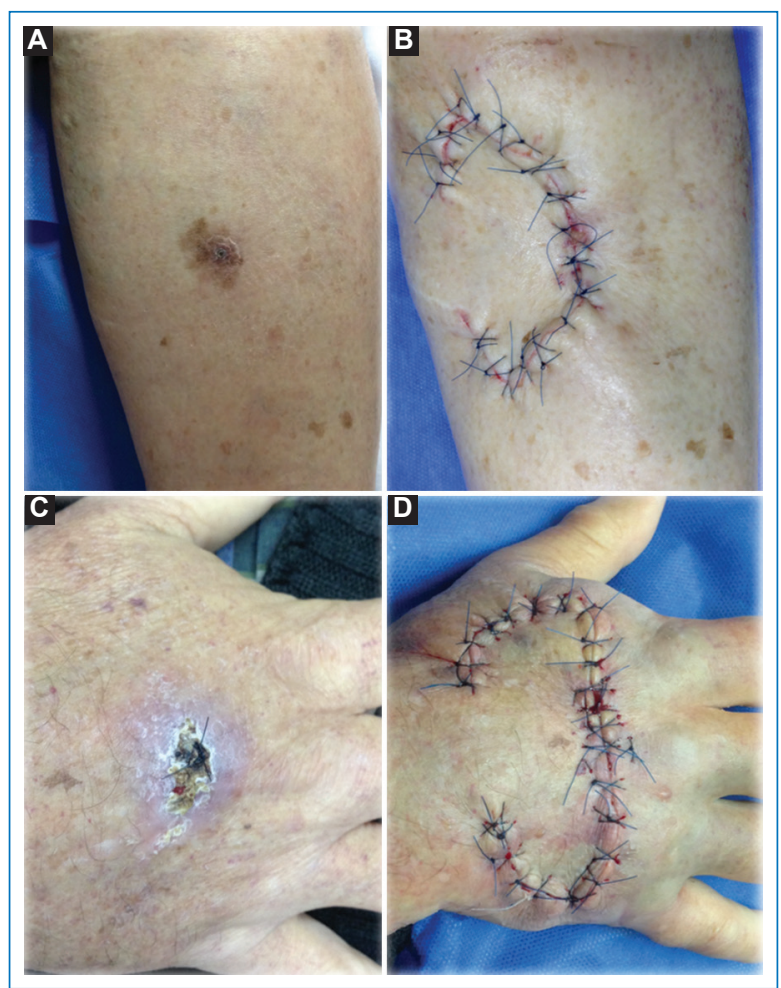

Figura 3. Defectos antes y después de la intervención quirúrgica. A: lentigo maligno en miembro inferior, antes de la escisión amplia con márgenes oncológicos.

B: reparación con CK modificado. C: carcinoma espinocelular infiltrante. D: reparación quirúrgica con CK modificado luego de la escisión con márgenes establecidos con mínima tensión.

\section{Posoperatorio (Fig. 3)}

Luego de terminar la intervención se realiza una curación compresiva para limitar el movimiento de la región del colgajo. Esto es de suma importancia para lograr una mejor sobrevida de éste y disminuir las complicaciones, como sangrado o dehiscencia de la herida. Los puntos se retiran después de 15 días.

\section{Puntos importantes}

- El diseño del colgajo se modifica de acuerdo con la zona y su angulación puede variar entre $90^{\circ}$ y $135^{\circ}$.

- El CK es un colgajo en isla en el cual la divulsión nunca debe realizarse por debajo del colgajo, ya que comprometería su sobrevida.

- Si el CK se aplica por debajo de la rodilla se debe indicar vendaje elastocompresivo al menos durante los siguientes 15 días.

- Para defectos de gran tamaño (> $50 \mathrm{~mm}$ ) puede efectuarse el doble CK para aprovechar al máximo la laxitud de los tejidos cercanos. Se diseña un segundo CK en forma opuesta al primero.

- La tunelización que aumenta la movilidad del colgajo es opcional.

\section{Discusión}

El CK es un colgajo en isla fasciocutáneo propuesto de manera primaria para la corrección de defectos infrapatelares y permite el cierre en un acto quirúrgico único (defecto y zona donante) sin requerir autoinjertos cutáneos ${ }^{4,7,8}$. La reconstrucción de grandes defectos con escaso volumen y falta de laxitud de piel obliga a utilizar colgajos libres, técnicamente difíciles y con tasa elevada de fracasos. La mayor parte de las veces se opta por opciones que producen excesiva tensión y 
cuidados posoperatorios difíciles, además de que son poco adheribles y costosos para el paciente.

La experiencia de los autores incluye cohortes prospectivas locales con diagnósticos de carcinoma espinocelular con distintos grados de diferenciación, carcinomas basocelulares y lentigos malignos en sitios con posibilidades reducidas de cierre (nulas de cierre primario), que de modo inicial hacían presumir defectos sobre $2 \mathrm{~cm}$ e indicación de cirugía de Mohs. En estos casos, la ejecución de la técnica en cuatro pasos del CK (diseño, divulsión y tunelización, cierre de puntos de mayor tensión y puntos en $\mathrm{X}$ para el cierre final, graficados en la Fig. 2), en algunos con modificación de la angulación para adaptarse a la superficie convexa (Fig. 1 C-D), permitió un cierre satisfactorio, resultados clínicos y cosméticos más que aceptables, y nulos efectos adversos mayores notificados luego se la intervención.

El CK ha demostrado sistemáticamente tener un rendimiento confiable, excelente capacidad de cobertura, complicaciones infrecuentes, rápida ejecución, mínima morbilidad (ideal para entornos de intervención con anestesia local) y buen resultado estético ${ }^{3,9,10-13}$. El diseño debe respetarse para garantizar la funcionalidad y la sobrevida, en los casos en que la divulsión y la tunelización son fundamentales, con objeto de disminuir la tensión y probabilidades de dehiscencia y ayudar a la movilidad y versatilidad en superficies mecánicamente dinámicas, de forma respectiva ${ }^{3,5,9,12}$.

El propósito de este artículo es considerar el CK como herramienta útil en la reconstrucción locorregional.

\section{Conclusión}

La corrección de defectos amplios que se escapan a la magnitud de la corrección locorregional típica ha abierto la necesidad de incorporar microcirugía y el desarrollo de colgajos libres. El CK es un colgajo que permite la reparación de defectos posquirúrgicos en lugares donde el cierre primario no es una opción viable. Es un colgajo en isla fasciocutáneo irrigado por las perforantes situadas por debajo del pedículo ancho y grueso de acuerdo con el diseño $3,7,9,10,13,14$.

El CK es un colgajo fiable, de fácil ejecución, con un bajo porcentaje de complicaciones que se reducen al mínimo al respetar el diseño y la técnica quirúrgica (distribución de la tensión, pedículo adecuado e inmovilización).

Puesto que es un colgajo, mantiene la similitud en el tipo de piel y coloración, lo que proporciona buenos resultados cosméticos. Debe considerarse como opción en defectos primarios mayores en los que el cierre simple no puede ejecutarse $e^{6,9,11-13}$.

\section{Agradecimientos}

Los autores agradecen al equipo del Centro de Cáncer de Piel y Cirugía Micrográfica de Mohs, Servicio de Dermatología del Hospital Italiano de Buenos Aires.

\section{Financiamiento}

Servicio de Dermatología del Hospital Italiano de Buenos Aires.

\section{Conflictos de intereses}

Los autores declaran no tener conflicto de intereses.

\section{Responsabilidades éticas}

Protección de personas y animales. Los autores declaran que para esta investigación no se han realizado experimentos en seres humanos ni en animales.

Confidencialidad de los datos. Los autores declaran que han seguido los protocolos de su centro de trabajo sobre la publicación de datos de pacientes.

Derecho a la privacidad y consentimiento informado. Los autores declaran que en este artículo no aparecen datos de pacientes.

\section{Bibliografía}

1. Behan FC. The keystone design perforator island flap in reconstructive surgery. ANZ J Surg. 2003;73:112-20.

2. Aragón-Miguel R, Gutiérrez-Pascual M, Sánchez-Gilo A, Sanz-Bueno J, Vicente-Martin FJ. Aplicación del colgajo de keystone en dermatología. Experiencia clínica en 18 pacientes. Actas Dermo-Sifiliográficas. 2018109(6):515-520

3. Gómez OJ, Barón OI, Peñarredonda ML. Keystone flap: overcoming paradigms. Plast Reconstr Surg Glob Open. 2019;7(3):e2126.

4. Huang J, Yu N, Long X, Wang X. A systematic review of the keystone design perforator island flap in lower extremity defects. Medicine (Baltimore). 2017;96(21):e6842.

5. Moncrieff MD, Thompson JF, Stretch JR. Extended experience and modifications in the design and concepts of the keystone design island flap. J Plast Reconstr Aesthet Surg. 2010;63:1359-63.

6. Abraham JT, Saint-Cyr M. Keystone and pedicle perforator flaps in reconstructive surgery: new modifications and applications. Clin Plast Surg. 2017 Apr;44(2):385-402.

7. Behan F. Evolution of the fasciocutaneous island flap leading to the keystone flap principle in lower limb reconstruction. ANZ J Surg. 2008;78:116-7

8. Colorado-Cogolludo L, Conde-Taboada A, González-Guerra E, Fueyo-Casado A, López-Bran E. Keystone flap used on the legs: case series. Clin Exp Dermatol. 2019;44(6):691-693

9. Mohan AT, Sur YJ, Zhu L, Morsy M, Wu PS, Moran SL, et al. The concepts of propeller, perforator, keystone, and other local flaps and their role in the evolution of reconstruction. Plast Reconstr Surg. 2016; 138(4):710e-29e. 
Med Cutan Iber Lat Am. 2021;49(3)

10. Darrach $H$, Kokosis G, Bridgham K, Stone JP, Lange JR, Sacks JM Comparison of keystone flaps and skin grafts for oncologic reconstruction: a retrospective review. J Surg Oncol. 2019;119(7): 843-849.

11. Yoon CS, Kim HB, Kim YK, Kim H, Kim KN. Relaxed skin tension line-oriented keystone-designed perforator island flaps considering the facial aesthetic unit concept for the coverage of small to moderate facia defects. Medicine (Baltimore). 2019;98(3):e14167.
12. Dobbs TD, Jovic TH, Jessop ZM, Kyle A, Hutchings HA, Whitaker IS. Objective and patient-reported assessments of skin grafts and keystone flaps-a pilot retrospective cohort study. Plast Reconstr Surg Glob Open. 201816;6(11):e2024.

13. Lee JW, Kim CW, Park TH. Customized reconstruction with modified keystone flaps. J Dermatol. 2018;45(7):844-849.

14. Jackson IT. The keystone design perforator island flap in reconstructive surgery. ANZ J Surg. 2003;73:261. 